\title{
REPRODUCTIVE PHENOLOGICAL PATTERNS OF CERRADO PLANT SPECIES AT THE PÉ-DE-GIGANTE RESERVE (SANTA RITA DO PASSA QUATRO, SP, BRAZIL): A COMPARISON BETWEEN THE HERBACEOUS AND WOODY FLORAS
}

\author{
BATALHA, M. A. and MANTOVANI, W. \\ Instituto de Biociências, Universidade de São Paulo, C.P. 11461, CEP 05422-970, São Paulo, SP, Brazil \\ Correspondence to: Instituto de Biociências, Universidade de São Paulo, C.P. 11461, CEP 05422-970, São Paulo, \\ SP, Brazil, e-mail: marcobat@uol.com.br \\ Received October 1, 1998 - Accepted November 16, 1998 - Distributed February 28, 2000
}

(With 6 figures)

\begin{abstract}
A natural vegetation area, with 1269 ha, composed mainly by cerrado, located at Santa Rita do Passa Quatro Municipality, São Paulo State, southeastern Brazil (21 ³6-39'S, 47³6-38’ W), was studied. From September 1995 to February 1997, a floristic survey was carried out, when all vascular plant species at reproductive stage were collected. The flowering and fruiting patterns of the community were studied, comparing the herbaceous and the woody species. In the herbaceous component, 239 species were found and, in the woody one, 108 species. The woody species flowered mainly at the beginning of the rainy season, while the herbaceous ones produced flowers generally at the end of that season. The proportion of anemo and autochorous species was greater in the herbaceous component. The zoochorous ones, on the contrary, were more frequent in the woody component. At the dry season, when their dispersion is more efficient, the proportion of anemo and autochorous species producing fruits was higher. During the rainy season, when their fruits become attractive for longer time, the zoochorous species fruited more intensely.
\end{abstract}

Key words: cerrado, savanna, phenology, Pé-de-Gigante, São Paulo, Brazil.

\section{RESUMO}

\section{Padrões fenológicos reprodutivos das espécies de um cerrado disjunto: comparação} entre as floras herbáceo-subarbustiva e arbustivo-arbórea

Foi estudada uma área de vegetação natural, de 1269 ha, composta principalmente por cerrado, situada no munícipio de Santa Rita do Passa Quatro, estado de São Paulo (21 ${ }^{\circ} 36-39^{\prime}$ S, 47 $36-38^{\prime}$ W). Entre setembro de 1995 e fevereiro de 1997, realizou-se um levantamento florístico, em que foram amostradas as espécies vasculares de plantas em fase fértil. A partir dos dados desse levantamento, estudaram-se os padrões de floração e frutificação da comunidade, comparando as espécies herbáceosubarbustivas com as arbustivo-arbóreas. No componente herbáceo-subarbustivo, foram encontradas 239 espécies e, no componente arbustivo-arbóreo, 108 espécies. As espécies arbustivo-arbóreas floresceram principalmente no início da estação chuvosa, enquanto as herbáceo-subarbustivas produziram flores, de modo geral, apenas no final da estação úmida. A proporção de espécies anemo e autocóricas foi maior no componente herbáceo-subarbustivo, ao contrário das zoocóricas, mais freqüentes no componente arbustivo-arbóreo. Na estação seca, quando sua dispersão é mais eficiente, a proporção de espécies anemo e autocóricas em frutificação foi maior. Já durante a estação chuvosa, as espécies zoocóricas frutificaram com maior intensidade, uma vez que seus frutos carnosos podem se manter atraentes por mais tempo.

Palavras-chave: cerrado, savana, fenologia, Pé-de-Gigante, São Paulo, Brasil. 


\section{INTRODUCTION}

Savannas are tropical and subtropical formations where the grass layer is continuous, interrupted by shrubs and trees in varying proportions, and where the main growth patterns are closely associated with alternating wet and dry seasons (Bourlière \& Hadley, 1983). In tropical savannas, the temporal patterns in growth and reproduction are linked to the climatic seasonality (Williams et al., 1997).

The so-called "cerrado" occupies approximately 2 millions $\mathrm{km}^{2}$ of the Brazilian territory (Ratter et al., 1992). It refers to several structural types, from grasslands to tall woodlands, most of which fit the definition of tropical savannas (Sarmiento, 1983).

Coutinho (1978), in his "woodland-ecotonegrassland" concept of cerrado, stated that there are in this vegetation type two floras, the herbaceous and the woody ones, which are perfectly distinct and antagonic.

The cerrado species, like those of other savannas, present periodic variations concerning flower and fruit production that may represent adaptations to biotic and abiotic factors (Schaik et al., 1993), determined by intrinsic genetic characters (Salisbury \& Ross, 1992).

The relation between the phenological patterns of the cerrado plant species and seasonality has already been discussed by Warming (1892). Since this pioneer study, several papers discuss some aspects of the cerrado species phenology (Barradas, 1972; Coutinho, 1977, 1982; Aoki \& Santos, 1980; Barros \& Caldas, 1980; Ribeiro et al., 1982; Gottsberger \& Silberbauer-Gottsberger, 1983; Oliveira \& Sazima, 1990; Oliveira \& Moreira, 1992; Miranda, 1995), but few of them do it at the community level (Mantovani \& Martins, 1988; Batalha et al., 1997).

Scholes \& Archer (1997) hypothesized that the climate seasonal pattern of tropical savannas, with alternating warm dry seasons with hot wet seasons, provides a potential axis of niche separation by phenology for the herbaceous and the woody components.

The aim of this study was to observe the reproductive phenological patterns of the plant species in a disjunct cerrado area at the community level, comparing the herbaceous and the woody floras.

\section{MATERIAL AND METHODS}

This study was carried out at the Pé-deGigante Reserve, at Santa Rita do Passa Quatro Municipality, São Paulo State, between $21^{\circ} 36-39^{\prime}$ 'S and $47^{\circ} 36-38^{\prime} \mathrm{W}$, under Köppen's Cwag' climate, at 660 to $730 \mathrm{~m}$ high, on Red-Yellow Latosol (Pivello et al., 1998). Its name ("Pé-de-Gigante" or "Giant's foot") was given after a foot-shaped geomorphological formation decurrent in the Paulicéia Stream drainage. The study area has 1,269 ha, 1,060 of which constitute the Reserve. According to Brazilian legislation, it's classified as an ARIE ("Área de Relevante Interesse Ecológico" or "Area of Relevant Ecological Interest"). The main vegetation type in the Reserve is the cerrado, covering $98 \%$ of the total area. A more detailed characterization of the study area can be found in Pivello et al. (1998).

The flowering and fruiting patterns of the cerrado species were analysed from data obtained in the floristic survey carried out from September 1995 to February 1997 (Batalha \& Mantovani, submitted). The botanical material at reproductive stage was collected with the walking-and-gathering method. The collected exsicata were deposited at São Paulo State Botanical Institute herbarium (SP), with doubles at São Paulo State Forestry Institute (SPSF) and Biosciences Institute of São Paulo University (SPF) herbaria.

The species at reproductive stage were observed whether they were flowering or fruiting. The observations were simply qualitative: for example, on a certain month, if at least one individual of a determined species was found producing flowers, the species was considered to be in its flowering period.

These informations were compared with climatic data collected from 1986 to 1995, at the Santa Rita do Passa Quatro meteorological station $\left(21^{\circ} 43^{\prime} 09^{\prime \prime} \mathrm{S}\right.$ and $\left.47^{\circ} 28^{\prime} 22^{\prime \prime} \mathrm{W}\right)$, from which a climatic diagram following Walter (1986) was constructed.

Pteridophytes were classified following Tryon $\&$ Tryon (1982), while angiosperms were included in families according to Cronquist (1988). The species were classified in life forms in accordance with Raunkiaer's system adapted by MuellerDombois \& Ellenberg (1974). The dispersion syndromes were classified according to Pijl (1972). 
The chamaephytes, epiphytes, geophytes, hemicryptophytes, lianas, vascular parasites, vascular hemi-parasites and therophytes species were considered as belonging to the herbaceous component, while the phanerophytes species as belonging to the woody one.

The flowering and fruiting periods were related to climatic variations and diaspore dispersion syndrome, comparing the herbaceous and the woody floras.

\section{RESULTS}

The climatic diagram (Fig. 1) shows that the dry period of the year lies between June and August, and the wet one between September and May. Mean annual rainfall and mean monthly temperature are, respectively, $1499 \mathrm{~mm}$ and $21.5^{\circ} \mathrm{C}$.

During the floristic survey, 239 herbaceous species (Table 1), belonging to 57 families, and 108 woody species (Table 2), representing 44 families, were found at reproductive stage.
The proportion of flowering herbaceous species was lower on August, increased till February, and decreased till August (Fig. 2). The proportion of flowering woody species peaked on September, and remained high until December. The lowest proportions of flowering species in this component were found from March to August. In the herbaceous component, the flower production was more intense at the end of the rainy season, while in the woody component it was concentrated at the beginning of this season.

The fruit production presented a similar pattern than the flower production, but slightly delayed (Fig. 3). The proportion of fruiting herbaceous species was lower on August, when it started to rise, reaching its peak on April, and then falling till August again. In the woody component, the lowest proportion of fruiting species was found also on August, when it quickly increased, peaking on November. From December to February, the proportions remained high, when it decreased quickly till August.

Santa Rita do Passa Quatro, SP (21ㄴ3'09"S, 4728'22"W, 749 m high)

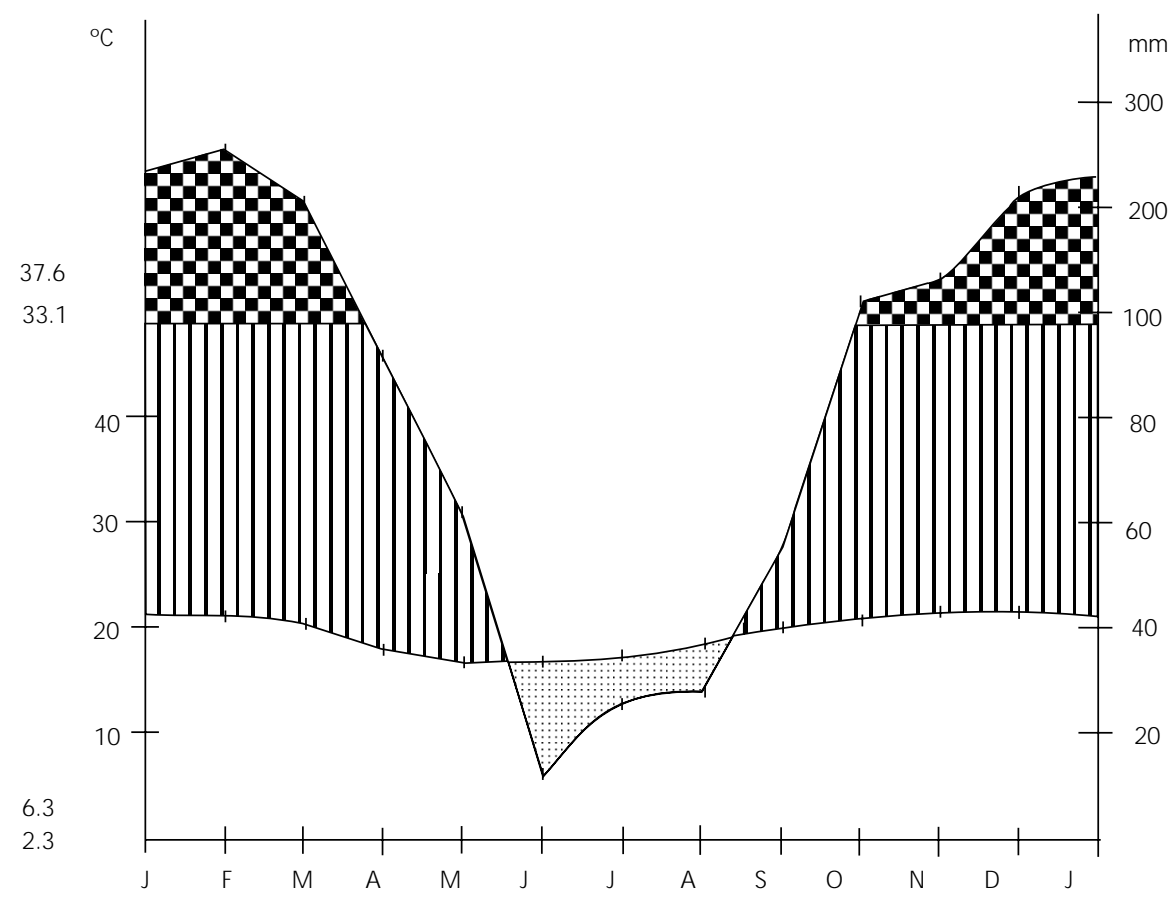

Fig. 1 - Climatic diagram following Walter (1986), constructed from data obtained at DAEE C4-107 meteorological station,

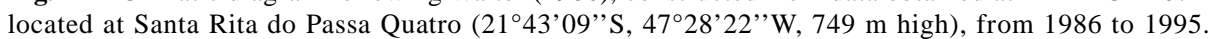




\section{TABLE 1}

List of collected herbaceous species at the Pé-de-Gigante Reserve, Santa Rita do Passa Quatro, SP (213639'S, 47 36-38'W). Disp. = dispersal syndrome, ANE = anemochorous, AUT $=$ autochorous, $\mathrm{ZOO}=$ zoochorous; fl. per. $=$ spores production or flowering period, $1=$ january, $2=$ february, ..., $11=$ november and $12=$ december; fruiting per. $=$ fr. period, $1=$ january, $2=$ february, $\ldots, 11=$ november and $12=$ december. The hyphen ( - ) represents continuity between months while the comma (, ) means interruption.

\begin{tabular}{|c|c|c|c|c|}
\hline Family & Species & disp. & fl. per. & fr. per. \\
\hline \multirow[t]{2}{*}{ Acanthaceae } & Hygrophila brasiliensis (Spr.) Lindau & AUT & 3 & 6 \\
\hline & Ruellia geminiflora H.B.K. & AUT & - & 4 \\
\hline \multirow[t]{4}{*}{ Amaranthaceae } & Alternanthera brasiliana (L.) Kuntze & AUT & $12-4$ & $2-5$ \\
\hline & Froelichia lanata (H.B.K.) Moq. & AUT & $11-4$ & $12-6$ \\
\hline & Gomphrena virgata Mart. & AUT & $10-5$ & $3-11$ \\
\hline & Pfaffia jubata Mart. & AUT & 10 & 10 \\
\hline Anacardiaceae & Anacardium humile A. St-Hil. & $\mathrm{ZOO}$ & 9 & $9-10$ \\
\hline \multirow[t]{2}{*}{ Annonaceae } & Annona coriacea Mart. & $\mathrm{ZOO}$ & $9-12$ & $9-12$ \\
\hline & A. dioica A. St-Hil. & $\mathrm{ZOO}$ & $11-12$ & 1 \\
\hline Apiaceae & Eryngium junceum Cham. \& Schltdl. & AUT & $12-1$ & 4 \\
\hline \multirow[t]{5}{*}{ Apocynaceae } & Forsteronia glabrescen Müll. Arg. & ANE & $10-3$ & $12-9$ \\
\hline & Mandevilla vellutina (Mart.) Woods. & ANE & $12-1$ & 1 \\
\hline & Odontadenia lutea (Vell.) Markgr. & ANE & $1-9$ & $5-10$ \\
\hline & Rhodocalyx rotundifolius Müll. Arg. & ANE & 12 & - \\
\hline & Temnadenia violacea (Vell.) Miers & ANE & $10-4$ & $10-5$ \\
\hline \multirow[t]{3}{*}{ Arecaceae } & Attalea geraensis Barb. Rodr. & $\mathrm{ZOO}$ & $4,10-11$ & - \\
\hline & Syagru flexuosa (Mart.) Becc. & $\mathrm{ZOO}$ & 9 & $9-11$ \\
\hline & S. petraea (Mart.) Becc. & $\mathrm{ZOO}$ & $3-7$ & 12 \\
\hline Aristolochiaceae & Aristolochia giberti Hook. & ANE & 2 & - \\
\hline \multirow[t]{5}{*}{ Asclepiadaceae } & Astephanus carassensi Malme & ANE & $4-5$ & - \\
\hline & Blepharodon nitidum (Vell.) J. Macbr. & ANE & $10-2$ & - \\
\hline & Ditassa acerosa Mart. & ANE & $3-6$ & - \\
\hline & D. nitida Fourn. & ANE & $4-6$ & 8 \\
\hline & Oxypetalum appendiculatum Mart. \& Zucc. & ANE & - & 9 \\
\hline \multirow[t]{14}{*}{ Asteraceae } & Acanthospermum australe (Loefl.) Kuntze & $\mathrm{ZOO}$ & - & 12 \\
\hline & Achyrocline satureoides (Lam.) A. DC. & AUT & $3-6$ & $4-10$ \\
\hline & Aspilia reflexa Baker & AUT & $11-1$ & $11-1$ \\
\hline & Baccharis dracunculifolia A. DC. & ANE & $12-4$ & $1-7$ \\
\hline & B. humilis Sch. Bip. & ANE & $11-4$ & $11-6$ \\
\hline & B. rufescens Spreng. & ANE & - & 9 \\
\hline & Bidens gardneri Baker & $\mathrm{ZOO}$ & $12-7$ & $12-8$ \\
\hline & Chaptalia integerrima (Vell.) Burk & ANE & $3-4$ & $3-4$ \\
\hline & Conyza canadensis (L.) Cronquist & ANE & $12-3$ & $12-3$ \\
\hline & Dasyphyllum sprengelianu (Gardner) Cabrera & ANE & $6-11$ & $6-11$ \\
\hline & Elephantopus biflora Less. & ANE & $2-5$ & $5-6$ \\
\hline & E. mollis $\mathrm{L}$. & ANE & $2-3$ & $6-9$ \\
\hline & Emilia coccinea (Simns) Sweet & ANE & $11-3$ & $11-3$ \\
\hline & Eremanthus sphaerocephalu Baker & ANE & 7 & $7-10$ \\
\hline
\end{tabular}


TABLE 1 (Continued)

\begin{tabular}{|c|c|c|c|c|}
\hline Family & Species & disp. & fl. per. & fr. per. \\
\hline \multirow[t]{26}{*}{ Asteraceae } & Eupatorium chlorolepsis Baker & ANE & 4 & - \\
\hline & E. maximiliani Schrad. & ANE & $4-7$ & $1-12$ \\
\hline & E. squalidum A. DC. & ANE & $3-6$ & $4-10$ \\
\hline & Gochnatia barrosii Cabrera & ANE & $8-10$ & $9-10$ \\
\hline & G. pulchra Cabrera & ANE & $2-8$ & $6-11$ \\
\hline & Kanimia oblongifolia Baker & ANE & $9-2$ & $9-2$ \\
\hline & Mikania cordifolia (L.) Willd. & ANE & $12-6$ & $2-10$ \\
\hline & Orthopappus angustifolius (Sw.) Gleason & ANE & $12-3$ & $2-11$ \\
\hline & Porophyllum angustissimum Gardner & ANE & $4-6$ & $6-9$ \\
\hline & P. ruderale (Jacq.) Cass. & ANE & 3 & 3 \\
\hline & Pterocaulon rugosum (Vahl) Malme & ANE & $3-4$ & $3-4$ \\
\hline & Trichogonia salviifolia Gardner & ANE & $12-8$ & $12-8$ \\
\hline & Vernonia apiculata Mart. & ANE & $2-4$ & $4-10$ \\
\hline & V. bardanoides Less. & ANE & $2-6$ & $2-10$ \\
\hline & V. cephalotes A. DC. & ANE & 8 & - \\
\hline & V. ferruginea Less. & ANE & 7 & - \\
\hline & V. herbacea (Vell.) Rusby & ANE & $1-3$ & $2-4$ \\
\hline & V. holosericea Mart. & ANE & $6-9$ & 9 \\
\hline & V. lappoides Baker & ANE & $3-4$ & $3-4$ \\
\hline & V. obtusata Less. & ANE & 6 & $6-7$ \\
\hline & V. onopordioides Baker & ANE & $3-5$ & $3-6$ \\
\hline & V. polyanthes (Spr.) Less. & ANE & $10-11$ & $10-7$ \\
\hline & V. rubriramea Mart. & ANE & $1-6$ & $6-10$ \\
\hline & V. scabra Pers. & ANE & 9 & $7-9$ \\
\hline & Viguiera discolo Baker & AUT & $1-5$ & $2-5$ \\
\hline & Wulffia stenoglossa A. DC. & AUT & $1-5$ & $2-5$ \\
\hline \multirow[t]{12}{*}{ Bignoniaceae } & Anemopaegma arvense (Vell.) Stellfeld & ANE & 一 & 10 \\
\hline & A. chamberlaynii (Simns) Bur. \& K. Schum. & ANE & - & 8 \\
\hline & Arrabidaea brachypoda (A. DC.) Bur. & ANE & $10-4$ & 4 \\
\hline & A. craterophora (A. DC.) Bur. & ANE & $1-5$ & 4-11 \\
\hline & A. florida A. DC. & ANE & $12-3$ & $7-9$ \\
\hline & Cremastus pulcher (Cham.) Bur. & ANE & $5-6$ & - \\
\hline & Distictella mansoana (A. DC.) Urban & ANE & $11-4$ & $1-12$ \\
\hline & Jacaranda caroba (Vell.) A. DC. & ANE & $3-10$ & $7-1$ \\
\hline & J. decurrens Cham. & ANE & $10-11$ & - \\
\hline & J. rufa Silva Manso & ANE & 12 & - \\
\hline & Memora peregrina (Miers.) Sandwith & ANE & $9-3$ & $9-3$ \\
\hline & Pyrostegia venusta (Ker) Bur. & ANE & $5-11$ & $7-12$ \\
\hline Bixaceae & Cochlospermum regium (Mart.) Pilg. & ANE & 9 & $9-11$ \\
\hline Boraginaceae & Cordia corymbosa (L.) G. Don. & ANE & 4 & - \\
\hline \multirow[t]{2}{*}{ Bromeliaceae } & Aechmea bromeliifolia (Rudge) Baker & ANE & 10 & 11 \\
\hline & Ananas ananassoides (Baker) L.B. Sm. & $\mathrm{ZOO}$ & $9-12$ & $9-5$ \\
\hline
\end{tabular}


TABLE 1 (Continued)

\begin{tabular}{|c|c|c|c|c|}
\hline Family & Species & disp. & fl. per. & fr. per. \\
\hline \multirow[t]{3}{*}{ Bromeliaceae } & Bromelia balansae Mez & $\mathrm{ZOO}$ & $9-12$ & $9-7$ \\
\hline & Dickia tuberosa (Vell.) Beer & ANE & 10 & 11 \\
\hline & Tillandsia geminiflora Brogn. & ANE & 11 & $4-5,9$ \\
\hline Cactaceae & Epyphyllum phyllanthus (L.) Haw. & $\mathrm{ZOO}$ & - & 11 \\
\hline \multirow[t]{6}{*}{ Caesalpiniaceae } & Chamaecrista campestris Irwin \& Barneby & AUT & $1-4$ & $1-4$ \\
\hline & C. cathartica (Mart.) Irwin \& Barneby & AUT & $12-6$ & 6 \\
\hline & C. debilis (Vogel) Irwin \& Barneby & AUT & $11-7$ & $1-12$ \\
\hline & C. desvauxii (Collad.) Killip & AUT & $1-7$ & $2-7$ \\
\hline & C. flexuosa (L.) Greene & AUT & $9-4$ & $2-9$ \\
\hline & C. rotundifolia (Pers.) Greene & AUT & 5 & $5-6$ \\
\hline Caryophyllaceae & Polycarpaea corymbosa (L.) Lam. & AUT & $12-4$ & $3-6$ \\
\hline Chrysobalanaceae & Parinari excelsa Sabine & $\mathrm{ZOO}$ & $9-12$ & $9-12$ \\
\hline Commelinaceae & Commelina erect $\mathrm{L}$. & AUT & $9-6$ & $9-6$ \\
\hline \multirow[t]{2}{*}{ Convolvulaceae } & Ipomoea procurrens C.F.W. Meissn. & AUT & $2-6$ & 一 \\
\hline & Jacquemontia tamnifolia (L.) Griseb. & AUT & $8-3$ & $8-3$ \\
\hline Cucurbitaceae & Cayaponia espelina (Cogn.) Silva Manso & $\mathrm{ZOO}$ & 11 & $12-2$ \\
\hline \multirow[t]{6}{*}{ Cyperaceae } & Bulbostylis hirtella (Schrad.) Urban & AUT & $9-5$ & $2-7$ \\
\hline & B. sphaerocephala (Boeck.) C.B. Clarke & AUT & $12-2$ & $2-6$ \\
\hline & Cyperus cayennensis (Lam.) Britton & AUT & $11-4$ & $1-5$ \\
\hline & C. diffusus Vahl & AUT & $11-5$ & $3-10$ \\
\hline & Rhynchospora exaltata Kunth & AUT & $5-1$ & $10-7$ \\
\hline & Scleria comosa (Nees) Steud. & AUT & $11-7$ & $12-8$ \\
\hline Dilleniaceae & Davilla rugosa A. St-Hil. & AUT & 4 & 4,11 \\
\hline \multirow[t]{8}{*}{ Euphorbiaceae } & Croton eriocladus Müll. Arg. & AUT & $12-5$ & $12-7$ \\
\hline & C. pohlianus Müll. Arg. & AUT & 11 & 4 \\
\hline & C. sclerocalyx Müll. Arg. & AUT & $12-2$ & $1-6$ \\
\hline & Manihot caerulescens Pohl & AUT & 11 & $12-4$ \\
\hline & M. tripartita Müll. Arg. & AUT & 2 & 11 \\
\hline & Phyllanthus orbiculatus Müll. Arg. & AUT & $11-6$ & $11-6$ \\
\hline & Sapium glandulatum (Vell.) Pax & $\mathrm{ZOO}$ & - & $1-2$ \\
\hline & Sebastiania serrulata Müll. Arg. & AUT & $10-3$ & $1-5$ \\
\hline \multirow[t]{11}{*}{ Fabaceae } & Aeschynomene marginata Benth. & ANE & $10-6$ & $1-12$ \\
\hline & Andira laurifolia Benth. & $\mathrm{ZOO}$ & $9-10$ & 12 \\
\hline & Centrosema venosum Mart. & AUT & 3 & - \\
\hline & Clitoria falcata Lam. & AUT & $2-3$ & $2-4$ \\
\hline & C. laurifolia Poir. & AUT & 11 & $12-3$ \\
\hline & Crotalaria vitellina Ker Gawl. & AUT & $1-3$ & $2-4$ \\
\hline & Deguelia nitidul (Benth.) Az.-Tozzi & AUT & $5-7$ & $6-8$ \\
\hline & Galactia decumbens (Benth.) Hassl. & AUT & 11 & - \\
\hline & G. grewiifolia (Benth.) Taub. & AUT & $10-4$ & $10-4$ \\
\hline & Indigofera suffruticosa Mill. & AUT & - & $5-7$ \\
\hline & Macroptilium gracile (Benth.) Urban & AUT & $3-6$ & 6 \\
\hline
\end{tabular}


TABLE 1 (Continued)

\begin{tabular}{|c|c|c|c|c|}
\hline Family & Species & disp. & fl. per. & fr. per. \\
\hline \multirow[t]{5}{*}{ Fabaceae } & Periandra mediterrane (Vell.) Taub. & AUT & $3-6$ & $5-6$ \\
\hline & Rhynchosia melanocarpa Grear & $\mathrm{ZOO}$ & $1-3$ & $1-3$ \\
\hline & Stylosanthes gracili H.B.K. & AUT & $10-7$ & $11-8$ \\
\hline & S. guianensis $\mathrm{Sw}$. & AUT & 5 & 5 \\
\hline & Zornia latifolia $\mathrm{Sm}$. & AUT & $11-4$ & $11-4$ \\
\hline Iridaceae & Trimezia juncifolia (K1.) Kunth & AUT & $2-4,10$ & - \\
\hline \multirow[t]{7}{*}{ Lamiaceae } & Hyptis brevipes Poit. & AUT & - & 5 \\
\hline & H. cana Pohl ex Benth. & AUT & 9 & 9 \\
\hline & H. eriophylla Pohl & AUT & $5-8$ & $6-9$ \\
\hline & H. mutabili (A. Rich.) Briq. & AUT & - & 5 \\
\hline & H. reticulata Mart. & AUT & $1-12$ & $1-12$ \\
\hline & H. rugosa Benth. & AUT & $2-9$ & $3-10$ \\
\hline & Peltodon tomentosus Pohl & AUT & $12-6$ & $2-9$ \\
\hline Lauraceae & Cassytha americana Nees & $\mathrm{ZOO}$ & - & 8 \\
\hline Liliaceae & Alstroemeria pulchella L. f. & AUT & $1-4$ & $3-4$ \\
\hline Loganiaceae & Strychnos bicolor Progel & $\mathrm{ZOO}$ & $11-2$ & $11-5$ \\
\hline Loranthaceae & Psittacanthus robustus Mart. & $\mathrm{ZOO}$ & $12-2$ & 10 \\
\hline Lythraceae & Cuphea carthaginensi (Jacq.) Macbr. & AUT & $11-3$ & $1-3$ \\
\hline \multirow[t]{9}{*}{ Malpighiaceae } & Banisteriopsis argyrophylla (A. Juss.) B. Gates & ANE & $4-5$ & - \\
\hline & B. campestris (A. Juss.) Little & ANE & $1-6$ & $2-10$ \\
\hline & B. laevifolia (A. Juss.) B. Gates & ANE & $12-5$ & $5-9$ \\
\hline & B. pubipetala (A. Juss.) Cuatrec. & ANE & $7-1$ & $9-1$ \\
\hline & B. stellaris (Griseb.) B. Gates & ANE & $12-7$ & $2-7$ \\
\hline & B. variabili B. Gates & ANE & 9 & $9-11$ \\
\hline & Heteropteris umbellat A. Juss. & ANE & $10-2$ & $11-2$ \\
\hline & Mascagnia cordifolia (A. Juss.) Griseb. & ANE & - & $10-11$ \\
\hline & Peixotoa tomentosa A. Juss. & ANE & $2-7$ & 7 \\
\hline \multirow[t]{7}{*}{ Malvaceae } & Pavonia communis A. St-Hil. & AUT & 2 & 2 \\
\hline & P. hexaphylla (S. Moore) Krapov. & AUT & $1-5$ & $2-5$ \\
\hline & Peltaea edouardii (Hochr.) Krapov. \& Cristóbal & AUT & $1-3$ & 3 \\
\hline & Sida glaziovii K. Schum. & AUT & 9 & $9-10$ \\
\hline & S. linifolia A. Juss. & AUT & $2-5$ & $3-5$ \\
\hline & S. rhombifolia L. & AUT & $1-3$ & 3 \\
\hline & S. urens $\mathrm{L}$. & AUT & 3 & 3 \\
\hline Melastomataceae & Miconia fallax DC. & $\mathrm{ZOO}$ & $2-10$ & $9-2$ \\
\hline Menispermaceae & Cissampelos ovalifolia Ruiz \& Pav. & $\mathrm{ZOO}$ & $10-2$ & 2 \\
\hline \multirow[t]{4}{*}{ Mimosaceae } & Mimosa debilis Humb. \& Bonpl. & $\mathrm{ZOO}$ & $1-3$ & $2-5$ \\
\hline & M. gracili Benth. & AUT & $1-2$ & - \\
\hline & M. pigra $\mathrm{L}$. & $\mathrm{ZOO}$ & 12 & - \\
\hline & M. xanthocentra Mart. & AUT & $9-2$ & $1-7$ \\
\hline \multirow[t]{2}{*}{ Myrtaceae } & Psidium australe Cambess. & $\mathrm{ZOO}$ & $9-10$ & $11-1$ \\
\hline & P. cinereum Mart. & $\mathrm{ZOO}$ & $10-11$ & $10-4$ \\
\hline
\end{tabular}


TABLE 1 (Continued)

\begin{tabular}{|c|c|c|c|c|}
\hline Family & Species & disp. & fl. per. & fr. per. \\
\hline \multirow[t]{2}{*}{ Orchidaceae } & Galeandra montana Barb. Rodr. & ANE & $11-4$ & 4 \\
\hline & Ionopsis paniculata Lindl. & ANE & 8 & - \\
\hline Oxalidaceae & Oxalis physocallyx Zucc. & AUT & $11-5$ & $12-5$ \\
\hline \multirow[t]{26}{*}{ Poaceae } & Andropogon leucostachy H.B.K. & AUT & 11 & $12-7$ \\
\hline & Aristida jubata (Arechav.) Herter & ANE & $3-5$ & $5-9$ \\
\hline & Axonopus barbigerus (Kunth) Hitchc. & $\mathrm{ZOO}$ & 2 & $\begin{array}{c}4-5,9- \\
10 \\
\end{array}$ \\
\hline & A. marginatus (Trin.) Chase & $\mathrm{ZOO}$ & $2-3$ & $4-5$ \\
\hline & Brachiaria decumbens Stapf & $\mathrm{ZOO}$ & $12-2$ & $1-8$ \\
\hline & Chloris barbata (L.) Sw. & $\mathrm{ZOO}$ & $1-2$ & 5 \\
\hline & Digitaria insularis (L.) Fedde & ANE & $12-2$ & - \\
\hline & Echinolaena inflexa (Poir.) Chase & $\mathrm{ZOO}$ & $12-4$ & $4-11$ \\
\hline & Eragrostis airoide Ness & $\mathrm{ZOO}$ & $12-2$ & $2-4$ \\
\hline & E. articulata (Schrank) Nees & $\mathrm{ZOO}$ & $1-4$ & 4 \\
\hline & E. maypurensis (H.B.K.) Steud. & $\mathrm{ZOO}$ & 4 & 4 \\
\hline & Gymnopogon foliosus (Willd.) Nees & ANE & $4-6$ & $5-9$ \\
\hline & Ichnanthus sericeus Hack. & $\mathrm{ZOO}$ & $9-5$ & $1-12$ \\
\hline & Loudetiopsis chrysothrix (Nees) Conert & $\mathrm{ZOO}$ & $2-3$ & $4-11$ \\
\hline & Melinis minutiflora $\mathrm{P}$. Beauv. & ANE & $6-7$ & $6-11$ \\
\hline & Panicum cayennensis Lam. & $\mathrm{ZOO}$ & - & $3-6$ \\
\hline & P. maximum Jacq. & $\mathrm{ZOO}$ & $4-5$ & $5-7$ \\
\hline & P. olyroides H.B.K. & $\mathrm{ZOO}$ & $11-3$ & $3-11$ \\
\hline & P. parvifolium Lam. & $\mathrm{ZOO}$ & $12-3$ & $3-10$ \\
\hline & P. procurrens Nees & $\mathrm{ZOO}$ & 3 & - \\
\hline & P. repens $\mathrm{L}$. & $\mathrm{ZOO}$ & $12-1$ & 6 \\
\hline & Rhynchelitrum repens (Nees) C.E. Hubb. & ANE & $5-2$ & $5-2$ \\
\hline & Schyzachirium condensatum (Kunth) Nees & ANE & 3 & $3-10$ \\
\hline & Setaria geniculata (L.) P. Beauv. & $\mathrm{ZOO}$ & - & 5 \\
\hline & Sporolobus indicus (L.) R. Br. & $\mathrm{ZOO}$ & $12-5$ & 5 \\
\hline & Tristachya leiostachya Nees & $\mathrm{ZOO}$ & $2-3$ & $5-7$ \\
\hline Polypodiaceae & Polypodium latipe Langsd. \& Fisch. & ANE & $1-12$ & - \\
\hline Polygalaceae & Securidaca tomentosa A. St-Hil. & ANE & $9-12$ & $10-12$ \\
\hline \multirow[t]{3}{*}{ Portulacaceae } & Portulaca hirsutissima Cambess. & AUT & $11-2$ & $12-2$ \\
\hline & P. mucronata Link & AUT & $11-12$ & - \\
\hline & Tallinum paniculatu (Jacq.) Gaertn. & AUT & $11-2$ & $11-4$ \\
\hline Pteridaceae & Adiantum fructuosum Spreng. & ANE & $9-3$ & - \\
\hline Rhamnaceae & Crumenaria polygaloides Reissek & ANE & - & 9 \\
\hline \multirow[t]{4}{*}{ Rubiaceae } & Alibertia sessili (Vell.) K. Schum. & $\mathrm{ZOO}$ & $6-9$ & $10-12$ \\
\hline & Borreria verticilata (L.) Mey. & AUT & $1-4$ & $2-4$ \\
\hline & B. warmingii K. Schum. & AUT & $12-1$ & $1-2$ \\
\hline & Coccocypselum lanceolatum (Ruiz \& Pav.) Pers. & $\mathrm{ZOO}$ & $1-2$ & $3-4$ \\
\hline
\end{tabular}


TABLE 1 (Continued)

\begin{tabular}{|c|c|c|c|c|}
\hline Family & Species & disp. & fl. per. & fr. per. \\
\hline \multirow[t]{9}{*}{ Rubiaceae } & Declieuxia fruticosa (Willd.) Kuntze & $\mathrm{ZOO}$ & $12-5$ & $12-7$ \\
\hline & Diodia schumanii Standl. & AUT & $11-1$ & $2-5$ \\
\hline & D. teres Walt. & AUT & 6 & $6-7$ \\
\hline & Palicourea coriacea (Cham.) K. Schum. & $\mathrm{ZOO}$ & $9-2$ & $11-4$ \\
\hline & Psychotria barbiflora A. DC. & $\mathrm{ZOO}$ & - & $4-6,12$ \\
\hline & P. capitata Ruiz \& Pav. & $\mathrm{ZOO}$ & $9-11$ & $9-5$ \\
\hline & P. deflexa A. DC. & $\mathrm{ZOO}$ & 12 & - \\
\hline & P. tricholoba Müll. Arg. & $\mathrm{ZOO}$ & $11-12$ & $4-5$ \\
\hline & Sabicea brasiliensis Wernham & $\mathrm{ZOO}$ & 6 & 6,11 \\
\hline \multirow[t]{6}{*}{ Sapindaceae } & Paullinia elegans Cambess & ANE & $7-10$ & - \\
\hline & Serjania erecta Radlk. & ANE & $11-2$ & $11-2$ \\
\hline & S. lethalis A. St-Hil. & ANE & 9 & $9-10$ \\
\hline & S. reticulata Cambess & ANE & $2-9$ & $5-10$ \\
\hline & Talisia angustifolia Raddi & $\mathrm{ZOO}$ & $9-11$ & 1 \\
\hline & Toulicia tomentosa Radlk. & ANE & $4-6$ & $5-10$ \\
\hline \multirow[t]{2}{*}{ Sapotaceae } & Pouteria subcaerulea Pierre ex Dubard & $\mathrm{ZOO}$ & $9-1$ & $12-2$ \\
\hline & Pradosia brevipes (Pierre) Penn. & $\mathrm{ZOO}$ & 10 & - \\
\hline Schizaeaceae & Anemia ferruginea H.B.K. & ANE & $2-4$ & - \\
\hline Scrophulariaceae & Buchnera lavandulacea Cham. \& Schltdl. & ANE & 4 & 4 \\
\hline Smilacaceae & Smilax cissoides Mart. ex Griseb & $\mathrm{ZOO}$ & $9-12$ & $11-2$ \\
\hline \multirow[t]{2}{*}{ Solanaceae } & Solanum lycocarpu A. St-Hil. & $\mathrm{ZOO}$ & $9-2$ & $1-2$ \\
\hline & S. palinacanthu Dunal & $\mathrm{ZOO}$ & $8-1$ & $8-3$ \\
\hline \multirow[t]{4}{*}{ Sterculiaceae } & Byttneria sagittifolia A. St-Hil. & AUT & $11-2$ & $2-5$ \\
\hline & Helicteres sacarolha A. St-Hil. & AUT & 3 & 一 \\
\hline & Waltheria americana $\mathrm{L}$. & AUT & $1-10$ & $1-10$ \\
\hline & W. communis $\mathrm{L}$. & AUT & $12-4$ & $2-4$ \\
\hline Turneraceae & Piriqueta rosea (Cambess.) Urban & AUT & $12-1$ & $12-1$ \\
\hline \multirow[t]{6}{*}{ Verbenaceae } & Lantana camara $\mathrm{L}$. & $\mathrm{ZOO}$ & $12-3$ & $12-3$ \\
\hline & L. fucata Lindl. & $\mathrm{ZOO}$ & 11 & 11 \\
\hline & Lippia lasiocalycina Cham. & AUT & $10-3$ & $11-3$ \\
\hline & L. lupulina Cham. & AUT & $9-11$ & $9-11$ \\
\hline & L. salviifolia Cham. & AUT & $1-4$ & $5-9$ \\
\hline & Stachytarpheta maximilliani Schauer & AUT & $12-3$ & $2-3$ \\
\hline \multirow[t]{2}{*}{ Vitaceae } & Cissus erosa Rich. & $\mathrm{ZOO}$ & $12-4$ & $2-4,9$ \\
\hline & C. inundata (Baker) Planch. & $\mathrm{ZOO}$ & $11-12$ & $11-12$ \\
\hline
\end{tabular}




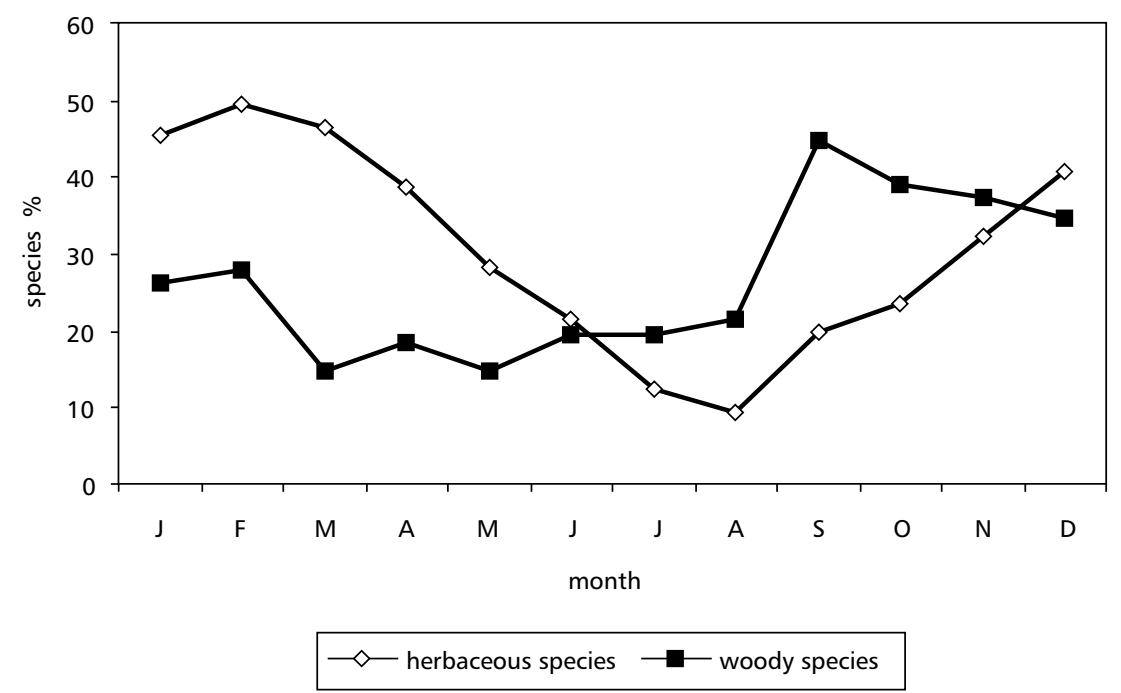

Fig. 2 - Percentage of cerrado species flowering through the year at the Pé-de-Gigante Reserve, Santa Rita do Passa Quatro, São Paulo $\left(21^{\circ} 36-39^{\prime} \mathrm{S}, 47^{\circ} 36-38^{\prime} \mathrm{W}\right)$.

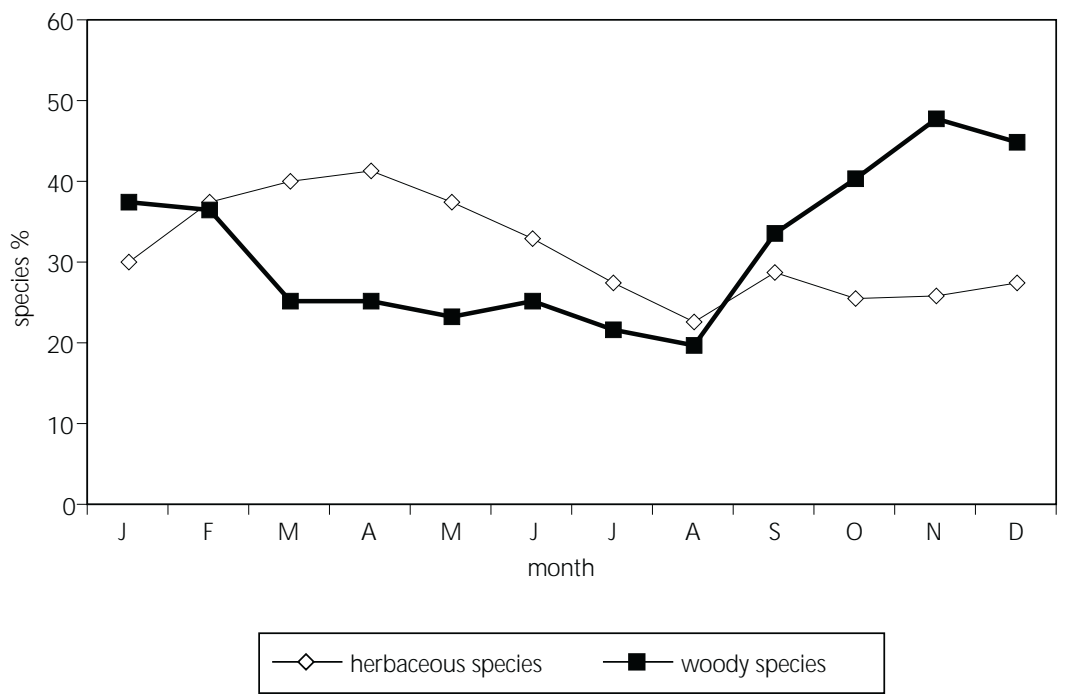

Fig. 3 - Percentage of cerrado species fruiting through the year at the Pé-de-Gigante Reserve, Santa Rita do Passa Quatro, São Paulo (2136-39'S, 47³6-38'W). 
TABLE 2

List of collected woody species at the Pé-de-Gigante Reserve, Santa Rita do Passa Quatro, SP (21³6-39'S, 47 36-38'W). Disp. = dispersal syndrome, $\mathrm{ANE}=$ anemochorous, $\mathrm{AUT}=$ autochorous, $\mathrm{ZOO}=$ zoochorous; fl. per. $=$ spores production or flowering period, $1=$ january, $2=$ february, $\ldots, 11=$ november and $12=$ december; fr. per. $=$ fruiting period, $1=$ january, $2=$ february, $\ldots, 11=$ november and $12=$ december. The hyphen ( - ) represents continuity between months while the comma ( , ) means interruption.

\begin{tabular}{|c|c|c|c|c|}
\hline Family & Species & disp. & fl. per. & fr. per. \\
\hline Anacardiaceae & Tapirira guianensis Aubl. & $\mathrm{ZOO}$ & $9-10$ & - \\
\hline \multirow[t]{3}{*}{ Annonaceae } & Annona crassiflora Mart. & $\mathrm{ZOO}$ & $11-12$ & $11-12$ \\
\hline & Duguetia furfuracea (A. St-Hil.) Benth. \& Hook. & $\mathrm{ZOO}$ & $11-7$ & $1-12$ \\
\hline & Xylopia aromatica A. St-Hil. & $\mathrm{ZOO}$ & $1-12$ & $1-12$ \\
\hline \multirow[t]{2}{*}{ Apocynaceae } & Hancornia speciosa Gomez & $\mathrm{ZOO}$ & $9-11$ & $9-11$ \\
\hline & Himatanthus obovatus (Müll. Arg.) Woods. & ANE & $12-6$ & - \\
\hline Araliaceae & Didymopanax vinosum (Cham. \& Schltdl.) Seem & $\mathrm{ZOO}$ & $2-11$ & $2-11$ \\
\hline \multirow[t]{2}{*}{ Arecaceae } & Butia paraguayensis (Barb. Rodr.) Bailey & $\mathrm{ZOO}$ & $4,10-12$ & - \\
\hline & Syagrus romanzoffiana (Cham.) Glass. & $\mathrm{ZOO}$ & $9-2$ & $2-11$ \\
\hline \multirow[t]{2}{*}{ Asteraceae } & Eremanthus erythropappa Sch. Bip. & ANE & $5-7$ & $7-9$ \\
\hline & Piptocarpha rotundifolia (Less.) Baker & ANE & $12-4$ & $1-12$ \\
\hline \multirow[t]{3}{*}{ Bignoniaceae } & Tabebuia aurea (Silva Manso) S. Moore. & ANE & $9-10$ & - \\
\hline & T. ochracea (Cham.) Standl. & ANE & 9 & - \\
\hline & Zeyhera montana Mart. & ANE & 4 & - \\
\hline Bombacaceae & Eriotheca gracilipes (K. Schum.) A. Robyns & ANE & $7-8$ & $9-12$ \\
\hline Burseraceae & Protium heptaphyllum (Aubl.) March & $\mathrm{ZOO}$ & $6-8$ & $8-2$ \\
\hline \multirow[t]{6}{*}{ Caesalpiniaceae } & Bauhinia rufa Steud. & AUT & $12-3$ & $1-12$ \\
\hline & Copaifera langsdorffii Desf. & $\mathrm{ZOO}$ & - & $9-6$ \\
\hline & Dyptichandra aurantiaca Tul. & ANE & $11-12$ & $1-11$ \\
\hline & Hymenaea stigonocarpa Mart. & $\mathrm{ZOO}$ & 1 & $7-10$ \\
\hline & Senna rugosa (G. Don.) Irwin \& Barneby & AUT & $2-6$ & $4-11$ \\
\hline & S. sylvestris (Vell.) Irwin \& Barneb & AUT & $2-3$ & 9 \\
\hline Caryocaraceae & Caryocar brasiliense Cambess. & $\mathrm{ZOO}$ & $9-11$ & $11-2$ \\
\hline Celastraceae & Plenckia populnea Reissek & ANE & 12 & - \\
\hline \multirow[t]{2}{*}{ Chrysobalanaceae } & Couepia grandiflora (Mart. \& Zucc.) Benth. & $\mathrm{ZOO}$ & $8-11$ & $10-2$ \\
\hline & Licania humilis Cham. \& Schltdl. & $\mathrm{ZOO}$ & $6-10$ & $10-12$ \\
\hline \multirow[t]{2}{*}{ Clusiaceae } & Kielmeyera rubriflora Cambess. & ANE & $4-6$ & - \\
\hline & K. variabilis Mart. & ANE & $12-2$ & $5-11$ \\
\hline Connaraceae & Rourea induta Planch. & $\mathrm{ZOO}$ & $8-12$ & $11-4$ \\
\hline Dilleniaceae & Davilla elliptica A. St-Hil. & AUT & $4-6$ & $6-1$ \\
\hline Ebenaceae & Diospyros hispida A. DC. & $\mathrm{ZOO}$ & - & $11-2$ \\
\hline \multirow[t]{4}{*}{ Erythroxylaceae } & Erythroxylum campestre A. St-Hil. & $\mathrm{ZOO}$ & 10 & $10-2$ \\
\hline & E. cuneifolium (Mart.) O.E. Schulz & $\mathrm{ZOO}$ & - & $10-11$ \\
\hline & E. suberosum A. St-Hil. & $\mathrm{ZOO}$ & $9-11$ & $10-2$ \\
\hline & E. tortuosum Mart. & $\mathrm{ZOO}$ & 11 & - \\
\hline \multirow[t]{2}{*}{ Fabaceae } & Acosmium subelegans (Mohl) Yakovlev & ANE & 11 & - \\
\hline & Andira anthelmia (Vell.) J. Macbr. & $\mathrm{ZOO}$ & 9 & 2 \\
\hline
\end{tabular}


TABLE 2 (Continued)

\begin{tabular}{|c|c|c|c|c|}
\hline Family & Species & disp. & fl. per. & fr. per. \\
\hline \multirow[t]{5}{*}{ Fabaceae } & Bowdichia virgilioides H.B.K. & ANE & $8-10$ & - \\
\hline & Dalbergia miscolobium Benth. & ANE & $9-2$ & $5-10$ \\
\hline & Machaerium acutifolium Vogel & ANE & $9-10$ & $9-6$ \\
\hline & Platypodium elegans Vogel & ANE & 12 & - \\
\hline & Pterodon pubescens Benth. & ANE & $9-10$ & $1-12$ \\
\hline \multirow[t]{2}{*}{ Flacourtiaceae } & Casearia grandiflora Cambess. & $\mathrm{ZOO}$ & $6-2$ & $9-2$ \\
\hline & C. sylvestris $\mathrm{Sw}$. & $\mathrm{ZOO}$ & $7-10$ & $9-10$ \\
\hline \multirow[t]{2}{*}{ Hippocrateaceae } & Peritassa campestris (Cambess.) A.C. Sm. & $\mathrm{ZOO}$ & $7-9$ & 11 \\
\hline & Tontelea micrantha (Mart.) A.C. Sm. & $\mathrm{ZOO}$ & $9-12$ & - \\
\hline \multirow[t]{2}{*}{ Lauraceae } & Ocotea corymbosa (Meiss.) Mez & $\mathrm{ZOO}$ & $11-12$ & $3-4$ \\
\hline & O. pulchella Mart. & $\mathrm{ZOO}$ & $1-4$ & $2-7$ \\
\hline Loganiaceae & Strychnos pseudoquina A. St-Hil. & $\mathrm{ZOO}$ & 10 & - \\
\hline Lythraceae & Lafoensia pacari A. St-Hil. & AUT & 4 & - \\
\hline \multirow[t]{2}{*}{ Malpighiaceae } & Byrsonima coccolobifolia A. Juss. & $\mathrm{ZOO}$ & $11-2$ & $12-2$ \\
\hline & B. crassa Nied. & $\mathrm{ZOO}$ & - & 2 \\
\hline \multirow[t]{3}{*}{ Malpighiaceae } & B. intermedia A. Juss. & $\mathrm{ZOO}$ & $9-5$ & $10-6$ \\
\hline & B. verbascifolia (L.) Rich ex A. Juss. & $\mathrm{ZOO}$ & $6-10$ & 11 \\
\hline & Heteropteris byrsonimifolia A. Juss. & ANE & $7-2$ & $9-4$ \\
\hline \multirow[t]{6}{*}{ Melastomataceae } & Leandra lacunosa Cogn. & ANE & $9-11$ & $11-7$ \\
\hline & Miconia albicans Triana & $\mathrm{ZOO}$ & $4-11$ & $6-1$ \\
\hline & M. ligustroides Naudin & $\mathrm{ZOO}$ & $11-6$ & $1-7$ \\
\hline & M. rubiginosa (Bonpl.) A. DC. & $\mathrm{ZOO}$ & $7-2$ & $9-6$ \\
\hline & M. stenostachya A. DC. & $\mathrm{ZOO}$ & $9-10$ & 11 \\
\hline & Tibouchina stenocarpa (A. DC.) Cogn. & ANE & $2-9$ & $5-10$ \\
\hline \multirow[t]{6}{*}{ Mimosaceae } & Anadenanthera falcata (Benth.) Speg. & AUT & $9-1$ & $1-12$ \\
\hline & A. peregrina (L.) Speg. & AUT & 2,9 & 4,12 \\
\hline & Dimorphandra mollis Benth. & AUT & $12-1$ & $1-9$ \\
\hline & Enterolobium gummiferum (Mart.) Macbr. & AUT & 10 & - \\
\hline & Plathymenia reticulata Benth. & ANE & - & 12 \\
\hline & Stryphnodendron polyphyllum Benth. & AUT & $12-1$ & $6-12$ \\
\hline Monimiaceae & Siparuna guianensis Aubl. & $\mathrm{ZOO}$ & $7-11$ & - \\
\hline \multirow[t]{2}{*}{ Moraceae } & Brosimum gaudichaudii Trècul & $\mathrm{ZOO}$ & $9-10$ & $9-11$ \\
\hline & Ficus citrifolia Mill. & $\mathrm{ZOO}$ & 12 & 12 \\
\hline Myristicaceae & Virola sebifera Aubl. & $\mathrm{ZOO}$ & $1-4$ & $5-11$ \\
\hline \multirow[t]{7}{*}{ Myrtaceae } & Campomanesia pubescens (A. DC.) O. Berg & $\mathrm{ZOO}$ & $9-11$ & $10-2$ \\
\hline & Eugenia aurata $\mathrm{O}$. Berg & $\mathrm{ZOO}$ & 11 & 3 \\
\hline & E. bimarginata A. DC. & $\mathrm{ZOO}$ & $5-7$ & $9-12$ \\
\hline & E. hiemalis Cambess. & $\mathrm{ZOO}$ & 5 & - \\
\hline & E. langsdorfii O. Berg & $\mathrm{ZOO}$ & 11 & 11 \\
\hline & E. livida O. Berg & $\mathrm{ZOO}$ & 8 & 12 \\
\hline & E. punicifolia (Kunth) A. DC. & $\mathrm{ZOO}$ & 1 & $2-4$ \\
\hline
\end{tabular}


TABLE 2 (Continued)

\begin{tabular}{|c|c|c|c|c|}
\hline Family & Species & disp. & fl. per. & fr. per. \\
\hline \multirow[t]{5}{*}{ Myrtaceae } & Myrcia bella Cambess. & $\mathrm{ZOO}$ & $9-12$ & $9-2$ \\
\hline & M. lasiantha A. DC. & $\mathrm{ZOO}$ & $9-11$ & $9-6$ \\
\hline & M. lingua O. Berg & $\mathrm{ZOO}$ & $9-12$ & $12-4$ \\
\hline & M. tomentosa (Aubl.) A. DC. & $\mathrm{ZOO}$ & $9-11$ & $11-12$ \\
\hline & M. uberavensis $\mathrm{O}$. Berg & $\mathrm{ZOO}$ & $7-12$ & $10-1$ \\
\hline \multirow[t]{2}{*}{ Nyctaginaceae } & Guapira noxia (Netto) Lund & $\mathrm{ZOO}$ & 10 & $10-11$ \\
\hline & Neea theifera Oerst. & $\mathrm{ZOO}$ & - & $11-1$ \\
\hline \multirow[t]{2}{*}{ Ochnaceae } & Ouratea castaneaefoli (A. DC.) Engl. & $\mathrm{ZOO}$ & 9 & - \\
\hline & O. spectabilis (Mart.) Engl. & $\mathrm{ZOO}$ & $6-10$ & $10-1$ \\
\hline Polygalaceae & Bredemeyera floribunda Willd. & AUT & $1-6$ & $5-10$ \\
\hline Proteaceae & Roupala montana Aubl. & AUT & 9 & - \\
\hline Rosaceae & Prunus sellowii $\mathrm{Sm}$. & $\mathrm{ZOO}$ & 12 & - \\
\hline \multirow[t]{5}{*}{ Rubiaceae } & Alibertia macrophylla K. Schum. & $\mathrm{ZOO}$ & 9 & $11-12$ \\
\hline & Amaioua guianensis Aubl. & $\mathrm{ZOO}$ & - & $2-5$ \\
\hline & Palicourea rigida H.B.K. & $\mathrm{ZOO}$ & $11-2$ & $1-6$ \\
\hline & Rudgea viburnoides (Cham.) Benth. & $\mathrm{ZOO}$ & - & 6 \\
\hline & Tocoyena formosa (Cham. \& Schltdl.) K. Schum. & $\mathrm{ZOO}$ & $11-2$ & $2-7$ \\
\hline Sapindaceae & Magonia pubescens A. St-Hil. & ANE & - & $1-3$ \\
\hline \multirow[t]{2}{*}{ Sapotaceae } & Pouteria ramiflora (Mart.) Radlk. & $\mathrm{ZOO}$ & $6-9$ & $12-2$ \\
\hline & P. torta (Mart.) Radlk. & $\mathrm{ZOO}$ & $6-9$ & $9-3$ \\
\hline Solanaceae & Solanum erianthum D. Don & $\mathrm{ZOO}$ & $10-6$ & 12 \\
\hline Styracaceae & Styrax ferrugineus Nees \& Mart. & $\mathrm{ZOO}$ & $2-9$ & $7-9$ \\
\hline Tiliaceae & Luehea divaricata Mart. & AUT & $9-10$ & $11-12$ \\
\hline Verbenaceae & Aegiphila lhotzkiana Cham. & $\mathrm{ZOO}$ & $11-12$ & - \\
\hline \multirow[t]{6}{*}{ Vochysiaceae } & Qualea dichotoma Warm. & ANE & - & 10 \\
\hline & Q. grandiflora Mart. & ANE & $9-2$ & $5-12$ \\
\hline & Q. multiflora Mart. & ANE & $12-2$ & - \\
\hline & Q. parviflora Mart. & ANE & $11-2$ & $9-4$ \\
\hline & Vochysia cinamomme Pohl & ANE & $2-4$ & $2-12$ \\
\hline & V. tucanorum Mart. & ANE & $12-2$ & 4 \\
\hline
\end{tabular}

Of the total herbaceous species, $38.07 \%$ are anemochorous, $36.98 \%$ are autochorous and $25.95 \%$ are zoochorous (Fig. 4). In the woody component, $25.92 \%$ of the species are anemochorous, $12.04 \%$, autochorous and the majority, $62.04 \%$, are zoochorous. Of the total anemochorous species, $76.47 \%$ belong to the first component, as well as $86.87 \%$ of the autochorous species. On the other side, the zoochorous species were distributed more evenly among the components, with $48.06 \%$ of them in the herba- ceous component and $51.94 \%$ in the woody one. In the herbaceous component, the anemochorous species fruited specially from June to October, the autochorous ones from February to June, and the zoochorous ones from November to May (Fig. 5). In the woody component, the anemochorous species fruited mainly from September to November, the autochorous ones from June to September, and the zoochorous ones from October to February (Fig. 6). 


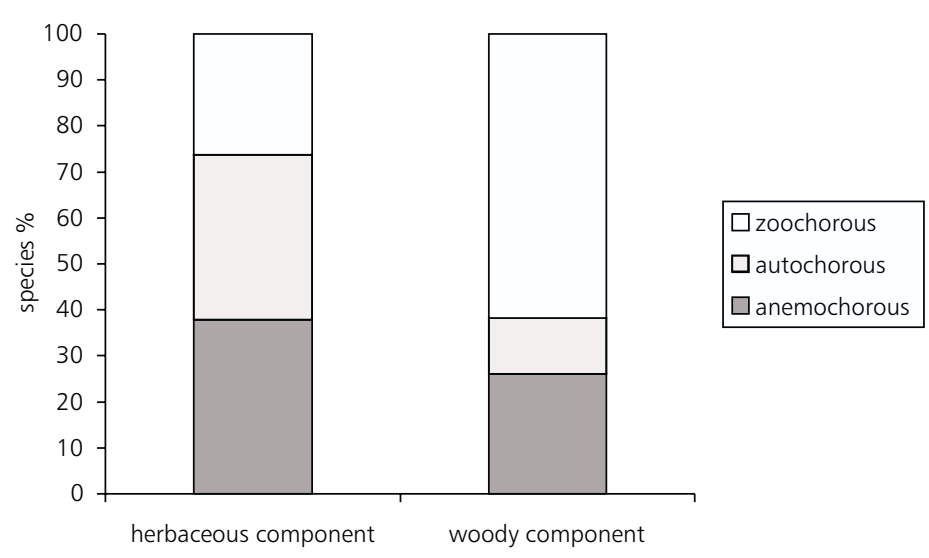

Fig. 4 - Percentual distribution of dispersion syndrome in the herbaceous and woody component of the cerrado species at the Pé-de-Gigante Reserve, Santa Rita do Passa Quatro, São Paulo (2136-39'S, 47³6-38'W).

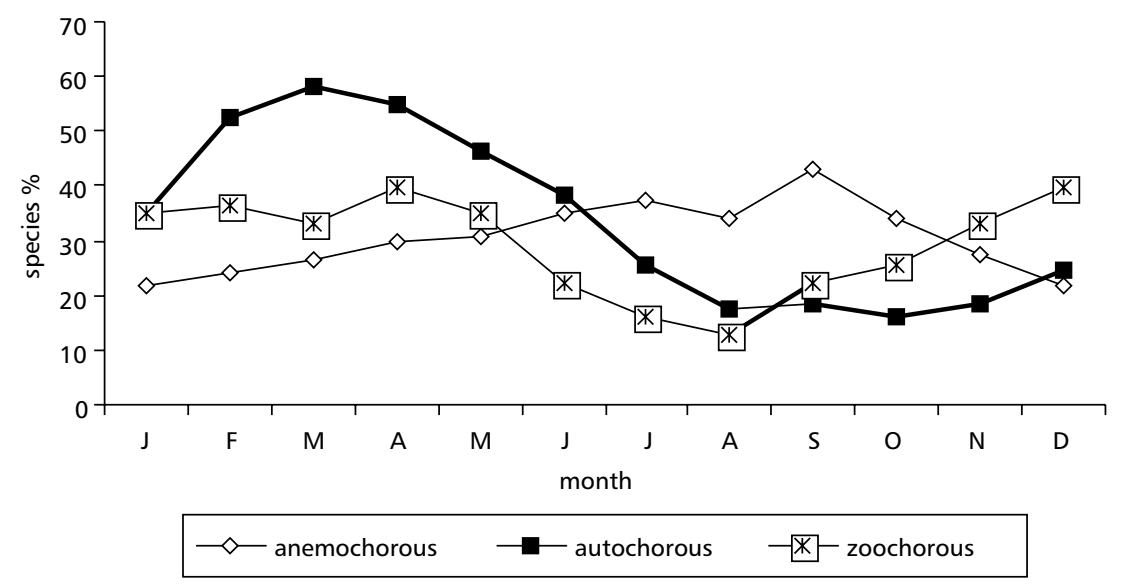

Fig. 5 - Percentual distribution of fruiting herbaceous species through the year at the Pé-de-Gigante Reserve, Santa Rita

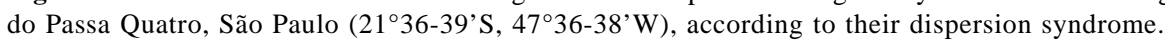

During the dry season, in both components, the proportions of fruiting anemo and autochorous species were higher than those of zoochorous species.

\section{DISCUSSION}

The flowering and fruiting patterns of the herbaceous species, late in the rainy season, are found in other tropical savannas too (Sarmiento \& Monasterio, 1983).

Tenório (1969) observed that the vegetative development of grasses allows carbohydrates accumulation that will be used in flowering and fruiting.

The carbohydrates accumulation of herbaceous species before flowering was also observed by Figueiredo \& Dietrich (1981). Sarmiento \& Monasterio (1983) hypothesized that this strategy would guarantee the reproduction in the safest period in respect of water availability: during the period of water shortage, the aerial biomass rapidly decays, and then as the rainy season progresses they gradually develop their shoots and reproductive structures, to reach the maximum growth rates during the reproductive phenophases that occur in the period with higher water availability. 


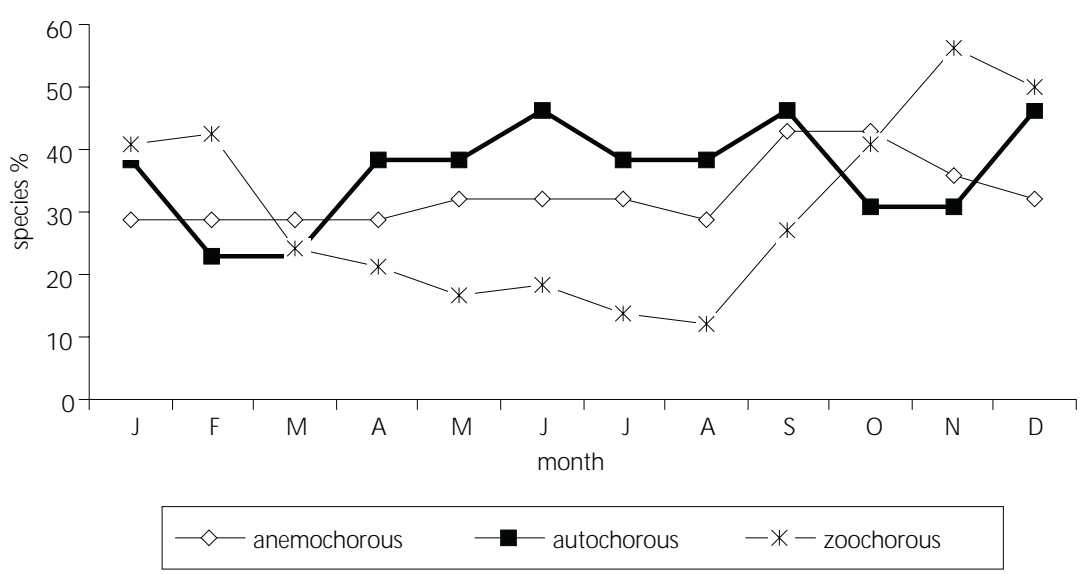

Fig. 6 - Percentual distribution of fruiting woody species through the year at the Pé-de-Gigante Reserve, Santa Rita do

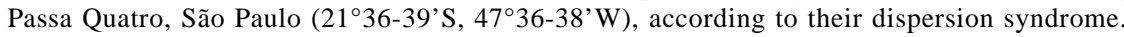

This strategy shows the importance of vegetative reproduction in mantaining the space occupied by the individual, since the underground storage reserves are utilized in flushing (Figueiredo \& Dietrich, 1981; Sarmiento \& Monasterio, 1983; Mantovani \& Martins, 1988).

The reproductive pattern found in the herbaceous species were quite different of those observed in the woody ones. The flowering period of the woody species was concentrated at the beginning of the rainy season. These results are in agreement with Mantovani \& Martins (1988) and Batalha et al. (1997). At lower latitudes, however, a distinct pattern was found: at Federal District (Aoki \& Santos, 1980) and at Pará State (Miranda, 1995), most species flower at the dry period of the year, what could be explained by the lower variations in temperature and day length through the year at these regions. Sarmiento \& Monasterio (1983) suggested that if trees and shrubs, through development of deep root systems, have water access during the whole year, what was observed by Rawitscher (1942) and Ferri (1944), there would be an advantage in reproducing during the period of water shortage, and leaving to the rainy season the function of storing reserves to support the dry season's activities. In accordance with Janzen (1980), the pollinating insects activity would be favoured at this time of the year, due to the lack of heavy rains that would damage the flowers and to the leaf fall that would make the flowers more visible.
Although several phenological patterns can be outlined within each component (Sarmiento \& Monasterio, 1983), a major distinction between them seems to exist. Scholes \& Archer (1997) stated that the coexistence of herbaceous and woody species in the savannas is a result of the interaction of several stresses and disturbances, acting differentially on each component and patchily in time and space. According to these authors, niches of herbaceous and woody species differ in both rooting depth and phenology, but there is more opportunity for phenological separation.

The diaspore syndrome proportions found at Santa Rita do Passa Quatro were close to those obtained by Batalha et al. (1997) at Pirassununga. Mantovani \& Martins (1988) found at Moji Guaçu a higher proportion of autochorous species and a lower one of zoochorous species. These authors, however, considered many grass species, here treated as zoochorous, autochorous. Gottsberger \& Silberbauer-Gottsberger (1983) found also a higher proportion of anemo and autochorous species in the herbaceous component and of zoochorous species in the woody one.

During the driest months, in both components, the proportion of fruiting anemochorous and autochorous species was higher than those of zoochorous ones, what was also found by Gottsberger \& Silberbauer-Gottsberger (1983), Mantovani \& Martins (1988), Miranda (1995) and Batalha et al. (1997). The anemochorous and autochorous fruits are generally dry and therefore 
their pericarp dehydrate in the drought, releasing the seeds. Augspurger \& Franson (1987) observed that, at seasonal climate areas, the anemochorous diaspore dispersion is more efficient at the dry season. The leaf fall observed mainly in the anemochorous species facilitates the diaspore dispersion as well (Matthes et al., 1988).

It could be noted for the zoochorous species that they fruited principally during the wettest and hottest period of the year, when their fleshy fruits can be kept attractive for a long time, showing the same pattern observed by Gottsberger \& Silberbauer-Gottsberger (1983), Mantovani \& Martins (1988), Miranda (1995) and Batalha et al. (1997).

Previous works with the woody component in other seasonal vegetation types (Frankie et al., 1974; Morellato et al., 1989) showed similar patterns, that is, flowering after first rainfalls, fructification of anemo and autochorous species at the dry season, and fructification of zoochorous species dispersed along the whole rainy season. Frankie et al. (1974), however, found more species flowering at the dry season than at the rainy one, as in other cerrado areas located at lower latitudes (Aoki \& Santos, 1980; Miranda, 1995).

\section{CONCLUSIONS}

The results found at the Pé-de-Gigante Reserve show the distinct flowering and fruiting patterns of the herbaceous and woody species. The herbaceous species flowered mainly at the end of the rainy season, while the woody ones at the beginning of this season. The proportion of anemo and autochorous species was higher in the herbaceous component, but the zoochorous species were more frequent in the woody component. During the dry season, the proportion of anemo and autochorous species producing fruits were higher, since their dispersion is facilitated at this time of the year. During the wet season, on the other hand, the zoochorous fruited more intensely, since their fruits can be kept attractive for a longer time.

These results, however, are only qualitative and therefore cannot provide secure answers to the questions raised. To accept or reject the hypothesis of niche separation by phenology for the herbaceous and the woody components, quantitative and experimental work is needed.
Anyway, the results here presented can be taken as guidelines for some phenological patterns of the cerrado plant species, principally at the community level, where few works have been carried out.

Acknowledgments - We are grateful to Paulo César Fernandes for field assistance and to FAPESP for the financial support conceded to the first author (process 95/4290-3).

\section{REFERENCES}

AOKI, H. \& SANTOS, J. R., 1980, Estudo da vegetação de cerrado na área do Distrito Federal, a partir de dados orbitais. MSc. Dissertation. Instituto Nacional de Pesquisas Espaciais.

AUGSPURGER, C. K. \& FRANSON, S. E., 1987, Wind dispersal of artificial fruits varying in mass, area and morphology. Ecol., 68: 27-42.

BARRADAS, M. M., 1972, Informações sobre a floração, frutificação e dispersão do piqui Caryocar brasiliense Camb. (Caryocaraceae). Ciênc. Cult., 24: 1063-1068.

BARROS, M. A. G. E. \& CALDAS, L. S., 1980, Acompanhamento de eventos fenológicos apresentados por cinco gêneros nativos do cerrado (Brasília, DF). Brasil Flor, 10: 7-14.

BATALHA, M. A., ARAGAKI, S. \& MANTOVANI, W., 1997, Variações fenológicas das espécies do cerrado em Emas (Pirassununga, SP). Acta Bot. Bras., 11: 61-78.

BATALHA, M. A. \& MANTOVANI, W., submitted, Floristic composition of the Pé-de-Gigante Reserve (Santa Rita do Passa Quatro, SP, Brazil). Rev. Brasil. Bot.

BOURLIÈRE, F. \& HADLEY, M., 1983, Present-day savannas: an overview. In: D. W. Goodall (ed.), Ecosystems of the world - tropical savannas. Elsevier, Amsterdam, pp. 1-17.

COUTINHO, L. M., 1977, Aspectos ecológicos do fogo no cerrado. II. As queimadas e a dispersão em algumas espécies anemocóricas do estrato herbáceo-subarbustivo. Bolm. Bot. Univ. S. Paulo, 5: 57-64.

COUTINHO, L. M., 1978, O conceito do cerrado. Rev. Brasil. Bot., 1: 17-23.

COUTINHO, L. M., 1982, Ecological effect of fire in Brazilian cerrado. In: B. J. Huntley \& B. H. Walker (eds.), Ecology of tropical savannas. Berlin, Springer-Verlag, pp. 273-291.

CRONQUIST, A, 1988, The evolution and classification of flowering plants. New York Botanical Garden, New York.

FERRI, M. G., 1944, Transpiração de plantas permanentes dos "cerrados". Bol. Fac. Filos. Ciênc. Letr. Univ. S. Paulo, Botânica, 4: 155-224.

FIGUEIREDO, R. C. L. \& DIETRICH, S. M. C., 1981 , Variações estacionais nos compostos de reserva e no metabolismo do xilopódio de Ocimum nudicaule Benth. var. anisifolia Giul. (Labiatae). Rev. Brasil. Bot., 4: 73-82. 
FRANKIE, G. W., BAKER, H. G. \& OPLER, P. A., 1974, Comparative phenological studies of trees in tropical wet and dry forest in the lowlands Costa Rica. J. Ecol., 62: 881-919.

GOTTSBERGER, G. \& SILBERBAUER-GOTTSBERGER, I., 1983, Dispersal and distribution in the cerrado vegetation of Brazil. Sonderbd. Naturwiss. Ver. Hemburg, 7: 315-352.

JANZEN, D. H., 1980, Ecologia vegetal nos trópicos. EPU e EDUSP, São Paulo.

MANTOVANI, W. \& MARTINS, F. R., 1988, Variações fenológicas das espécies do cerrado da Reserva Biológica de Moji Guaçu, Estado de São Paulo. Rev. Brasil. Bot., 11: 101-112.

MATTHES, L. A. F., LEITÃO-FILHO, H. F. de \& MARTINS, F. R., 1988, Bosque dos Jequitibás (Campinas, SP): Composição florística e estrutura do estrato arbóreo. In: Congr. Soc. Bot. S. Paulo 5, Botucatu. Anais. Soc. Bot. S. Paulo, Campinas, pp. 55-76.

MIRANDA, I. S., 1995, Fenologia do estrato arbóreo de uma comunidade de Alter-do-Chão, PA. Rev. Brasil. Bot., 18(2): 235-240.

MORELLATO, L. P., RODRIGUES, R. R., LEITÃO-FILHO, H. F. de \& JOLY, C. A., 1989, Estudo comparativo da fenologia de espécies arbóreas de floresta de altitude e floresta mesófila semidecídua na Serra do Japi, Jundiaí, São Paulo. Rev. Brasil. Bot., 12: 85-98.

MUELLER-DOMBOIS, D. \& ELLENBERG, H., 1974, Aims and methods of vegetation ecology. John Willey \& Sons, New York.

OLIVEIRA, P. E. \& MOREIRA, A. G., 1992, Anemocoria em espécies de cerrado e mata galeria de Brasília, DF. Rev. Brasil. Bot., 5: 163-174.

OLIVEIRA, P. E. \& SAZIMA, M., 1990, Pollination biology of two species of Kielmeyera (Guttiferae) form Brazilian cerrado vegetation. Plant Syst. Evol., 172: 35-49.

PIJL, L. van der, 1972, Principles of dispersion in higher plants. Springer-Verlag, Berlin.

PIVELLO, V. R., BITENCOURT, M. D., MANTOVANI, W., MESQUITA JR., H. N. de, BATALHA, M. A. \& SHIDA, C., 1998, Proposta de zoneamento ecológico para a reserva de cerrado Pé-de-Gigante (Santa Rita do Passa Quatro, SP). Braz. J. Ecol., 2: 108-118.
RATTER, J. A., RIBEIRO, J. F. \& BRIDGEWATER, S., 1992, The Brazilian cerrado vegetation and threats to its biodiversity. Ann. Bot., 80: 223-230.

RAWITSCHER, F., 1942, Algumas noções sobre a transpiração e o balanço de água de plantas brasileiras. Anais Acad. Bras. Ciênc., 14: 7-36.

RIBEIRO, J. F., GONZALES, M. I. \& OLIVEIRA, P. E., 1982, Aspectos fenológicos de espécies nativas de cerrado. In: XXXIII Congresso Nacional de Botânica, Teresina, 1981. Anais. Soc. Bot. Brasil, Teresina, pp. 181-198.

SALISBURY, F. B. \& ROSS, C. W., 1992, Plant physiology. Wadsworth, Belmont.

SARMIENTO, G., 1983, The savannas of tropical america. In: D. W. Goodall (ed.), Ecosystems of the world tropical savannas. Elsevier, Amsterdam, pp. 245-288.

SARMIENTO, G. \& MONASTERIO, M., 1983, Life forms and phenology. In: D. W. Goodall (ed.), Ecosystems of the world - tropical savannas. Elsevier, Amsterdam, pp. 79-108.

SCHAIK, C. P. van, TERBORGH, J. W. \& WRIGHT, S. J., 1993, The phenology of tropical forests: adaptive significance and consequences for primary consumers. Ann. Rev. Ecol. Syst., 24: 353-377.

SCHOLES, R. J. \& ARCHER, S. R., 1997, Tree-grass interactions in savannas. Ann. Rev. Ecol. Syst., 28: 517-544.

TENÓRIO, E. C., 1969, Fenologia de gramíneas. In: Congr. Soc. Bot. Bras. 20, Goiânia, 1969. Anais. Soc. Bot. Brasil, Goiânia, pp. 231-241.

TRYON, R. M. \& TRYON, A. F., 1982, Ferns and allied plants with special reference to Tropical America. Springer-Verlag, New York.

WALTER, H., 1986, Vegetação e zonas climáticas. EPU, São Paulo.

WARMING, E., 1892, Lagoa Santa, contribuição para a geografia fitobiológica. In: E. Warming \& M. G. Ferri, 1973, Lagoa Santa e a vegetação dos cerrados brasileiros. Itatiaia, Belo Horizonte, pp. 1-282.

WILLIAMS, R. J., MYERS, B. A., MULLER, W. J., DUFF, G. A. \& EAMUS, D., 1997, Leaf phenology of woody species in a north Australian tropical savanna. Ecol., 78(8): 2542-2558. 